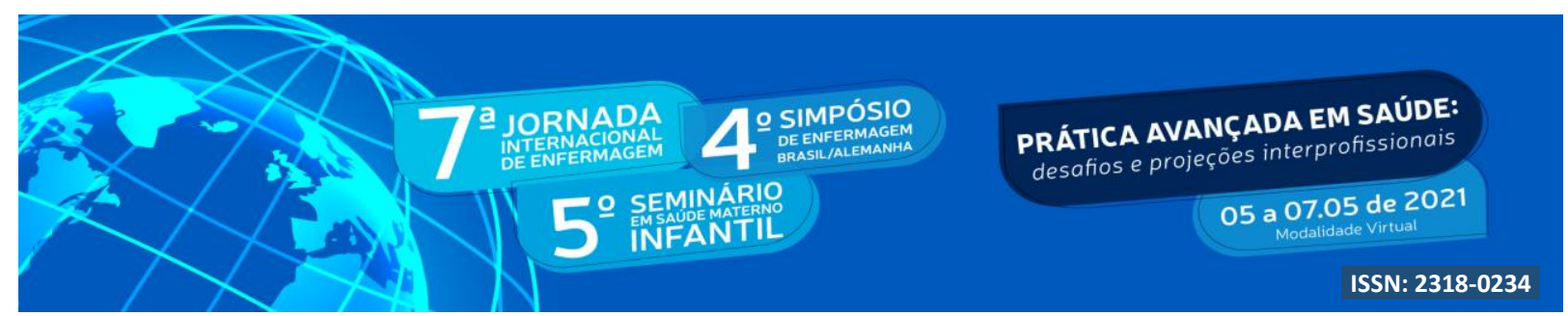

DOI: http://doi.org/10.48195/jie2021-125

\title{
CONCENTRAÇÃO SÉRICA E CONSUMO DE VITAMINA C EM PACIENTES HOSPITALIZADOS COM AVC ${ }^{1}$
}

\author{
Patricia Beche²; Mairin Schott ${ }^{3}$; Sérgio Roberto Mortari ${ }^{4}$; Elisângela Colpo ${ }^{5}$
}

\begin{abstract}
RESUMO
Objetivou-se analisar concentrações séricas e consumo alimentar da vitamina $\mathrm{C}$ em pacientes hospitalizados com Acidente Vascular Cerebral (AVC). Realizou-se coleta de dados como idade, gênero, tabagismo e etilismo, além de dados de consumo de vitamina $\mathrm{C}$ por meio do recordatório alimentar 24 horas e os níveis séricos foram determinados por Cromatografia à Líquido de Alta Eficiência (CLAE). Participaram do estudo 64 pacientes. Não foram observadas diferenças significativas dos níveis de vitamina $C$ sérica entre adultos e idosos $(P>0,05)$, mas os pacientes encontraram-se dentro dos valores de referência. $\mathrm{O}$ consumo de vitamina $\mathrm{C}$ se mostrou maior em mulheres $(77,7 \pm 62,3 \mathrm{mg})$, e os homens $(61,9 \pm 49,3 \mathrm{mg})$ apresentaram um consumo médio abaixo dos valores recomendados para ingestão. Conclui-se que os níveis séricos de vitamina $\mathrm{C}$ estão adequados, mas um consumo mais baixo foi observado nos homens. A vitamina $\mathrm{C}$ pode auxiliar no tratamento e prognóstico da doença.
\end{abstract}

Palavras-chave: Ácido Ascórbico; Humanos; Níveis séricos.

\begin{abstract}
The objective was to analyze serum concentrations and food consumption of vitamin $\mathrm{C}$ in patients hospitalized with stroke. Data were collected, such as age, gender, smoking and alcohol consumption, in addition to data on vitamin $\mathrm{C}$ consumption through the 24-hour food record and serum levels were determined by High Efficiency Liquid Chromatography (HPLC). 64 patients participated in the study. There were no significant differences in serum vitamin $\mathrm{C}$ levels between adults and the elderly $(\mathrm{P}>$ 0.05), but the patients were within the reference values. The consumption of vitamin $C$ was higher in women $(77.7 \pm 62.3 \mathrm{mg})$, and men $(61.9 \pm 49.3 \mathrm{mg})$ had an average consumption below the recommended values for ingestion. It is concluded that the serum levels of vitamin $\mathrm{C}$ are adequate, but a lower consumption was observed in men. Vitamin $\mathrm{C}$ can help in the treatment and prognosis of the disease.
\end{abstract}

Key Words: Ascorbic acid; Humans; Serum levels.

\footnotetext{
${ }^{1}$ Trabalho de Iniciação Científica (PROBIC/UFN).

${ }^{2}$ Estudante do Curso de Nutrição. UFN - Universidade Franciscana. E-mail: patricia.beche@ufn.edu.br

${ }^{3}$ Nutricionista pela Universidade Franciscana. Doutoranda em Ciências Biológicas: Bioquímica Toxicológica pela UFSM. E-mail: mairinschott@ hotmail.com

${ }^{4}$ Professor do Curso de Biomedicina e do Mestrado em Nanociências da Universidade Franciscana - UFN mortari@ufn.edu.br

${ }^{5}$ Professora do Curso de Nutrição e do Mestrado em Ciências e Saúde da Vida da Universidade Franciscana UFN.elicolpo@ufn.edu.br
} 


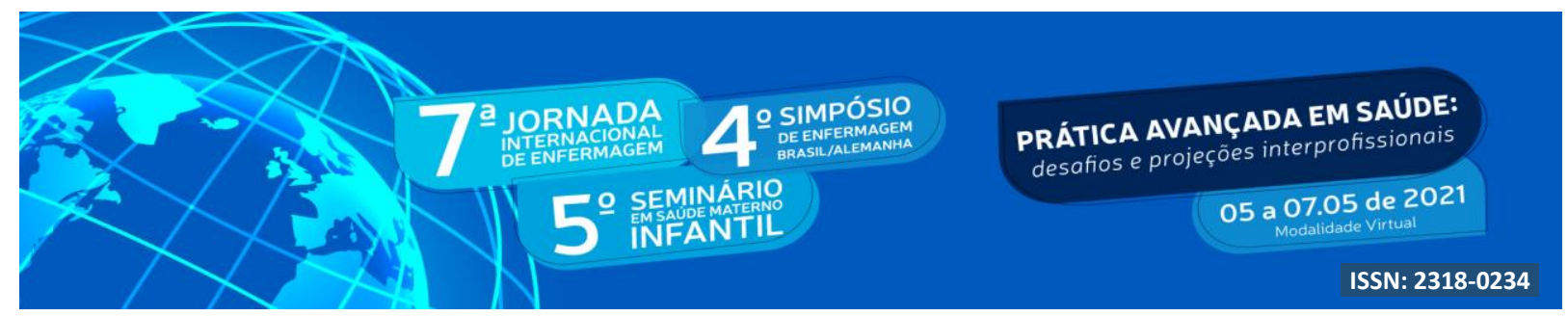

\section{INTRODUÇÃO}

O Acidente Vascular Cerebral (AVC) é definido como o surgimento de um déficit neurológico súbito causado por obstrução ou rompimento dos vasos sanguíneos do sistema nervoso central. O AVC pode ser isquêmico que acontece pela obstrução ou redução do fluxo sanguíneo em uma artéria causada por êmbolos ou trombos. Também pode ser hemorrágico causado pela ruptura espontânea de um vaso, com extravazamento de sangue para dentro das áreas extra vasculares do cérebro, ocasionando aneurisma ou trauma (REDE BRASIL, 2021).

O Acidente Vascular Cerebral é a segunda maior causa de morte no mundo, responsáveis por aproximadamente $11 \%$ do total de mortes. No ano de 2018, só no Brasil, foram registradas 176.273 internações por doenças cerebrovasculares (WHO, 2020; DATASUS, 2018). Um estudo realizado no ano de 2018 evidenciou que a maior porcentagem de mortes por AVC em geral ocorreu em indivíduos homens com 70 anos ou mais. O AVC isquêmico foi o tipo mais comum, sendo responsável por $61,8 \%$ destas mortes (DE SANTANA et al., 2018).

Em relação aos fatores de risco para Acidente Vascular Cerebral, as frequências de tabagismo e etilismo são altas, principalmente no sexo masculino, e podem aumentar as taxas de morbidade e mortalidade nesta população (BRUBACHER; MOSER; JORDAN, 2000).

Alguns estudos demostraram que valores baixos de vitamina $C$ sérica ou plasmática tem associação com o aumento da incidência de acidente vascular cerebral e também com a mortalidade por AVC (KURL et al., 2002).

A vitamina C ou Ácido Ascórbico tem papel fundamental nas vias fisiológicas, pois age protegendo biomoléculas (proteínas, lipídios e DNA) por meio das suas propriedades antioxidantes, assim ajuda a preservar as funções e estrutura do tecido, tem papel na geração de energia, está envolvida na síntese de hormônios. Além de ter um papel na determinação do prognóstico de pacientes criticamente enfermos (SHARMA et al., 2019).

A avaliação do consumo de vitamina $C$, segue as recomendações da necessidade média estimada (EAR, do inglês estimated average requirement) onde a recomendação de vitamina $\mathrm{C}$ para homens com idade entre 19 a >70 anos é $75 \mathrm{mg}$ e para mulheres da mesma faixa etária 60 mg. Já em relação aos níveis máximos de ingestão toleráveis (UL, do inglês tolerable upper intakes levels), é de até 2000 mg para homens e mulheres (PADOVANI et al., 


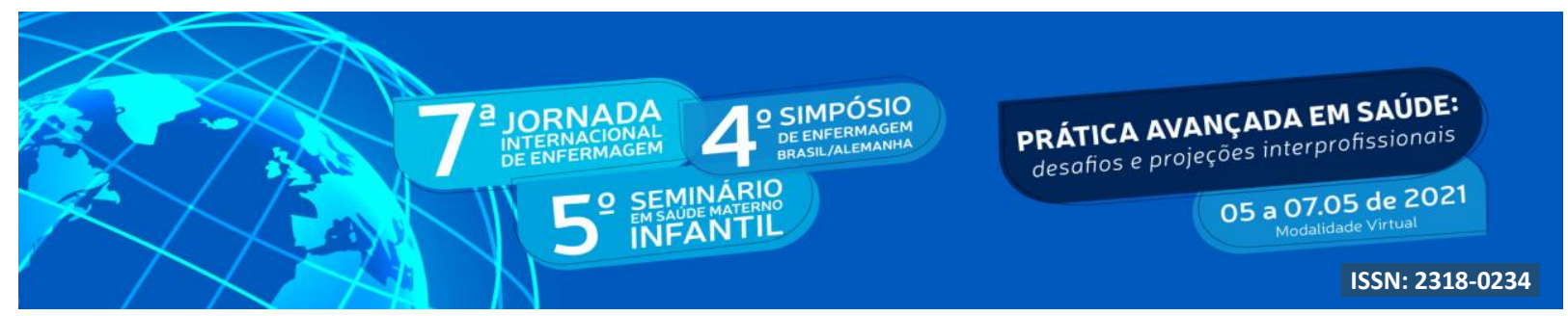

2006).

Se sabe que indivíduos fumantes e com faixa etária entre 60 a 96 anos necessitam de ingestão diária mais alta de vitamina $\mathrm{C}$, aproximando-se de $35 \mathrm{mg}$ além do recomendado, para equilibrar os níveis séricos, se comparados com indivíduos não-fumantes entre 15 a 60 anos (BRUBACHER; MOSER; JORDAN, 2000; PIRES; GAGLIARDI; GORZONI, 2004).

Em relação aos valores de vitamina $\mathrm{C}$ sérica, são considerados valores maiores ou iguais a $28 \mu \mathrm{mol} / \mathrm{L}$ como normais, e valores abaixo de $28 \mu \mathrm{mol} / \mathrm{L}$ são definidos em depleção de vitamina C. Já os valores séricos abaixo de $11 \mu \mathrm{mol} / \mathrm{L}$ são definidos em deficiência de vitamina C (JOHNSTON; CORTE, 1999).

Sendo assim o estudo justifica-se em relacionar os niveis séricos e de consumo alimentar da vitamina $\mathrm{C}$, visto que esta vitamina desempenha funções essenciais ao organismo, possuindo grande importância para o tratamento e prognóstico do paciente com AVC.

\section{OBJETIVO}

Analisar as concentrações séricas e o consumo alimentar de vitamina $\mathrm{C}$ em pacientes hospitalizados com AVC.

\section{METODOLOGIA}

O trabalho a seguir trata-se de um estudo transversal. Os dados da pesquisa foram coletados em um hospital público da região central do Rio Grande do Sul, durante os meses de agosto 2018 a agosto de 2019. A amostra foi por conveniência, composta por adultos e idosos hospitalizados com diagnóstico de AVC, de ambos os gêneros. O estudo foi aprovado pelo Comitê de Ética e Pesquisa e está registrado sob o número 1.579.090, conforme a Resolução n. 466/12 do Conselho Nacional de Saúde, referente a pesquisas com seres humanos. O termo de consentimento livre e esclarecido (TCLE) foi obtido por escrito de todos os participantes.

Dados de identificação pessoal dos pacientes como gênero, idade, tabagismo e etilismo foram coletados do prontuário. A avaliação dietética desses pacientes foi obtida em até 72 horas após a internação. Os parâmetros de consumo alimentar dos pacientes alimentados por 


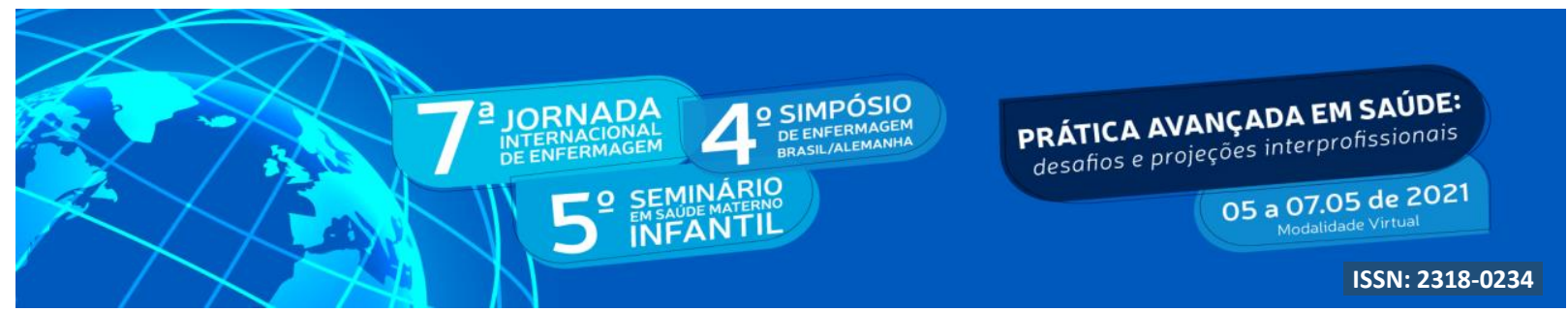

via oral foram avaliados pelo recordatório do consumo do dia após a internação dos pacientes.

Para acompanhar o consumo alimentar dos pacientes, o recordatório do consumo do dia anterior foi aplicado três vezes em dias não consecutivos durante a internação do paciente. Para calcular o recordatório de cada paciente, foi realizado o cadastro das fichas técnicas no programa Nutrisoft Brazil, em que foi possível quantificar a quantidade de vitamina $\mathrm{C}$ de cada paciente.

Para avaliar a ingestão dietética dos pacientes com indicação de Terapia Nutricional Enteral (TNE) foram verificadas as intercorrências da TNE (pausa para exames, obstrução da sonda, retirada da sonda e outras intercorrências) e o volume infundido da dieta enteral que o paciente estava recebendo. A partir da composição nutricional das dietas industrializadas disponíveis no hospital foram calculadas calorias, carboidrato, lipídio, proteína, vitamina C, selênio, zinco e cobre de cada paciente. As informações da composição nutricional de cada dieta enteral disponível no hospital, foram retirados dos respectivos rótulos contidos nas dietas.

Para realização da análise da vitamina $\mathrm{C}$ sérica as amostras de sangue foram adquiridas a partir da sobra dos tubos de coletas destinados para as análises sanguíneas dos pacientes durante a hospitalização sendo, posteriormente, armazenadas a $-80^{\circ} \mathrm{C}$. As amostras foram precipitadas com TCA 5\% (1:1), homogeneizadas com o auxílio do vórtex e centrifugadas à $3000 \mathrm{RPM}$ a $10^{\circ} \mathrm{C}$ por 10 minutos. A análise de vitamina $\mathrm{C}$ sérica foi realizada Cromatografia à Líquido de Alta Eficiência (CLAE) sendo utilizada uma coluna de

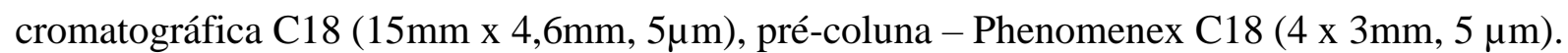

Posteriormente, os dados obtidos foram tabulados e armazenados em uma tabela no programa Excel. A análise estatística foi realizada no programa de Statistica versão 10.0, onde foi aplicado testes paramétrico (Test-T) ou não paramétrico (Mann-whitney ou KruskalWallis) de acordo com o teste de normalidade de Shapiro-Wilk. Os dados foram considerados estatisticamente significativos quando $\mathrm{p}<0,05$.

\section{RESULTADOS E DISCUSSÃO}

Participaram do estudo 64 pacientes hospitalizados com AVC. Dos participantes 57,8\% $(n=37)$ eram do gênero masculino 42,2\% $(n=27)$ do gênero feminino; 67,2\% ( $n=43)$ dos 


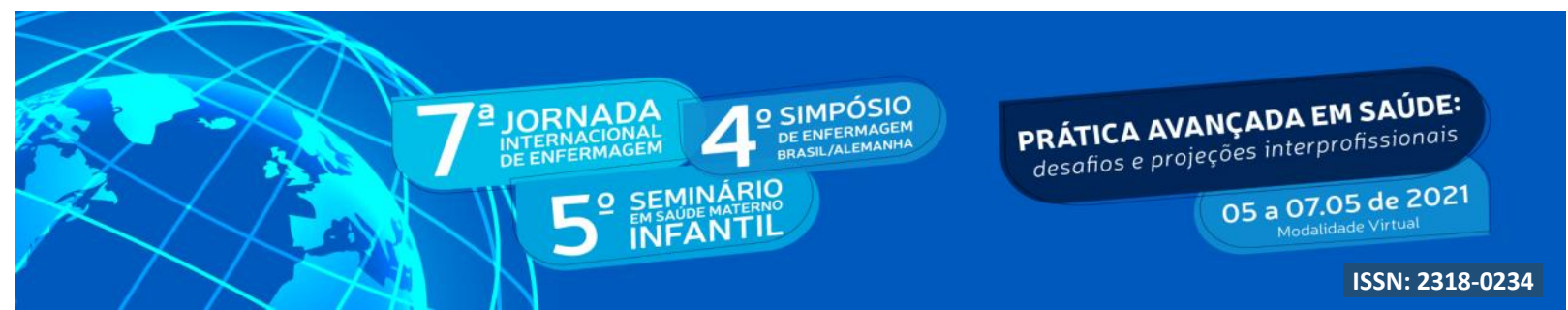

indivíduos estudados eram idosos, 29,7\% ( $n=19)$ eram tabagistas e 26,6\% $(n=17)$ etilistas. Em relação ao tipo de AVC, $72 \%(\mathrm{n}=46)$ dos pacientes tiveram AVC isquêmico e $28 \%$ $(\mathrm{n}=18)$ hemorrágico.

Se observa na Tabela 1 que não foram observadas diferenças significativas entre adultos e idosos $(\mathrm{P}>0,05)$. Vale destacar que os pacientes encontram-se dentro dos valores de referência (> $28 \mu \mathrm{mol} / \mathrm{L}$ ), conforme Johnston e Corte (1999). O estudo de Sánchez Moreno et al., (2004) mostou que pacientes com AVC, tiveram níveis plasmaticos de vitamina $\mathrm{C}$ mais baixos quando comparados a controles, embora a ingestão fosse semelhante, sugerindo que o AVC isquêmico possa reduzir os níveis de vitamina C.

Tabela 1: Níveis séricos vitamina $\mathrm{C}$ em pacientes hospitalizados com AVC.

\begin{tabular}{cccc}
\hline Idade & Vitamina $\mathbf{C}(\boldsymbol{\mu m o l} / \mathbf{L})$ & $\mathbf{p}$ & $\mathbf{n}$ \\
\hline Adulto $(<60$ anos $)$ & $310,0 \pm 220,7$ & $0,949^{2}$ & 21 \\
\hline Idoso $(>60$ anos $)$ & $306,7 \pm 179,0$ & & 43 \\
\hline
\end{tabular}

${ }^{1}$ Teste Não-paramétrico; ${ }^{2}$ Teste paramétrico

Conforme a Tabela 2, o consumo de vitamina $\mathrm{C}$ em pacientes com Acidente Vascular Cerebral, se mostrou maior em mulheres. Os homens apresentaram um consumo médio abaixo dos valores recomendados para ingestão diária. Em relação a faixa etária, o consumo foi maior em idosos, mas ambos resultados não obtiveram diferenças estatísticas significativas $(\mathrm{P}>0,05)$.

Em relação ao tabagismo, o consumo foi menor em pacientes ex-tabagistas quando comparados a tabagistas e não tabagistas, mas sem diferenças significativas. Quando realizada análise de correlação entre a vitamina $\mathrm{C}$ sérica e o seu consumo em pacientes hospitalizados com AVC não foram observadas diferenças estatísticas $(\mathrm{R}=0,11 ; \mathrm{P}=0,37)$.

Tabela 2: Consumo de vitamina $\mathrm{C}$ em pacientes hospitalizados com AVC.

\begin{tabular}{cccc}
\hline Sexo & Vitamina C $(\mathbf{m g})$ & $\mathbf{p}$ & $\mathbf{n}$ \\
\hline Masculino & $61,9 \pm 49,3$ & $0,342^{1}$ & 36 \\
\hline Feminino & $77,7 \pm 62,3$ & & 27 \\
\hline Idade & Vitamina C $(\mathbf{m g})$ & $\mathbf{p}$ & $\mathbf{n}$ \\
\hline Adulto $(<60$ anos $)$ & $61,1 \pm 58$ & $0,443^{2}$ & 21 \\
\hline Idoso (>60 anos) & $72,5 \pm 54,3$ & & 42 \\
\hline Tabagismo & Vitamina C $(\mathbf{m g})$ & $\mathbf{p}$ & $\mathbf{n}$ \\
\hline Tabagista & $68,3 \pm 42,9$ & $0,837^{1}$ & 18 \\
\hline Não tabagista & $72,4 \pm 61,4$ & & 29
\end{tabular}




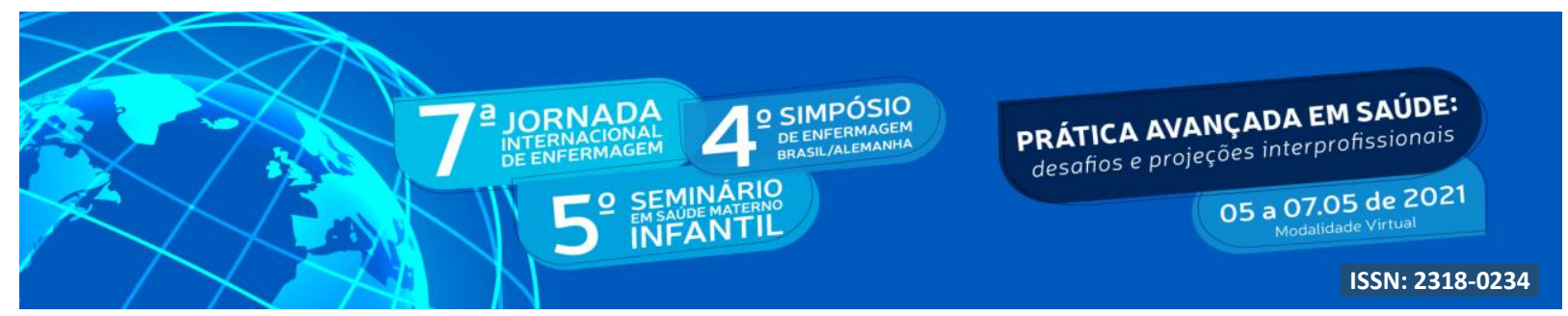

\begin{tabular}{cccc}
\hline Ex-tabagista & $62,4 \pm 58,8$ & & 16 \\
\hline Etilismo & Vitamina C $(\mathbf{m g})$ & $\mathbf{p}$ & $\mathbf{n}$ \\
\hline Etilista & $95,9 \pm 72,6$ & $0,159^{1}$ & 17 \\
\hline Não etilista & $57,6 \pm 45,6$ & & 38 \\
\hline Ex-etilista & $63,5 \pm 39,7$ & & 8 \\
\hline
\end{tabular}

${ }^{1}$ Teste Não-paramétrico; ${ }^{2}$ Teste paramétrico

A vitamina $\mathrm{C}$ atua como antioxidante, que apresenta vários papéis fisiológicos no corpo (DUARTE; LUNEC, 2005). Quando acontece um desequilíbrio do sistema de defesa antioxidante, podem ocorrer danos pela ação nociva das espécies reativas ao oxigênio. Esses danos podem incluir redução no sistema imunológico e aumento do risco de infecções (CLARKSON; THOMPSON, 2000). Por esse motivo é importante adequar o consumo de vitamina $\mathrm{C}$ em pacientes hopitalizados. Neste estudo, observamos que em média o consumo de vitamina $\mathrm{C}$ dos pacientes hospitalizados está dentro dos valores de referência. Do mesmo modo, os níveis séricos dos pacientes também estão dentro da normalidade (JOHNSTON; CORTE, 1999).

Uma limitação do traballho foi que o estudo aconteceu com pacientes hospitalizados com AVC com outros tipos de patologias, o que pode ter influenciado nos níveis séricos de vitamina $\mathrm{C}$, com diferenças bem importantes entre os pacientes, apresentando assim um desvio padrão muito alto. Além disso, o número pequeno de pacientes estudados podem ter influenciado no valor não significativo dos dados estatísticos.

\section{CONCLUSÃO}

Os dados do presente estudo mostram que os níveis séricos de vitamina $\mathrm{C}$ estão adequados, no entanto, observou-se um consumo médio baixo desta vitamina nos homens, conforme valores de recomendação diária. A vitamina $\mathrm{C}$ pode atuar como um fator protetor em pacientes com AVC, podendo auxiliar no tratamento e prognóstico da doença. Porém, mais estudos seriam necessários para investigar essa questão.

\section{REFERÊNCIAS}

Rede Brasil AVC. O que é o AVC?. Disponivel em: http://www.redebrasilavc.org.br/parapacientes-e-falimiares/o-que-e-avc/. Acesso: 23/03/2021. 


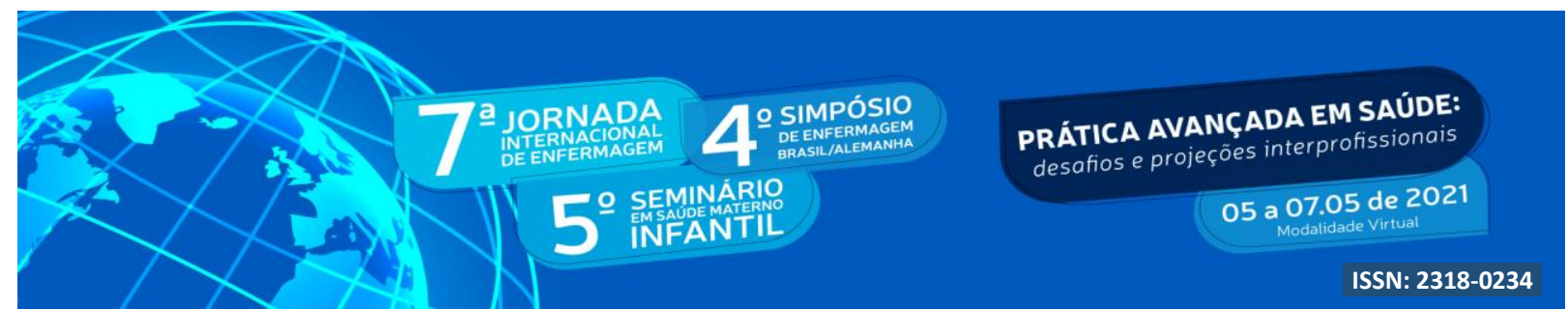

BRUBACHER, D.; MOSER, U.; JORDAN, P. Vitamin C concentrations in plasma as a function of intake: A meta-analysis. International Journal for Vitamin and Nutrition Research, [s. l.], v. 70, n. 5, p. 226-237, 2000.

CLARKSON, P. M; THOMPSON, H. S. Antioxidants: what role do they play in physical activity and health? The American Journal of Clinical Nutrition, v.72, n.2, p. 637-46, 2000 .

DATASUS. Morbidade Hospitalar do SUS- por local de internação- Brasil. p. CID-10 Acidente vascular cerebral isquêmico transitório e síndrome correl, Acidente vascular cerebral não especificado hemorrágico ou isquêmico, 2018.

DE SANTANA, Nathalia Matos et al. The burden of stroke in Brazil in 2016: An analysis of the Global Burden of Disease study findings 11 Medical and Health Sciences 1117 Public Health and Health Services. BMC Research Notes, [s. l.], v. 11, n. 1, p. 1-5, 2018.

DUARTE, T. L.; LUNEC, J. J. Review: when is an antioxidant not an antioxidant? A review of novel actions and reactions of vitamin C. Free Radical Research, v. 39, n. 7, p. 671-686, 2005.

JOHNSTON, CAROL S; CORTE, CORINNE. People with Marginal Vitamin C Status are at High Risk of Developing Vitamin C Deficiency. Journal of the American Dietetic Association, [s. l.], v. 99, n. 7, p. 854-856, 1999.

KURL, S. et al. Plasma vitamin C modifies the association between hypertension and risk of stroke. Stroke, [s. l.], v. 33, n. 6, p. 1568-1573, 2002.

PADOVANI, Renata Maria et al. Dietary reference intakes: Application of tables in nutritional studies. Revista de Nutricao, [s. l.], v. 19, n. 6, p. 741-760, 2006.

PIRES, Sueli Luciano; GAGLIARDI, Rubens José; GORZONI, Milton Luiz. Estudo das freqüências dos principais fatores de risco para acidente vascular cerebral isquêmico em idosos. Arquivos de Neuro-Psiquiatria, [s. l.], v. 62, n. 3b, p. 844-851, 2004.

SÁNCHEZ-MORENO, Concepción et al. Decreased Levels of Plasma Vitamin C and Increased Concentrations of Inflammatory and Oxidative Stress Markers after Stroke. Stroke, [s. l.], v. 35, n. 1, p. 163-168, 2004.

SHARMA, Yogesh et al. Vitamin C deficiency in Australian hospitalised patients: an observational study. Internal Medicine Journal, [s. l.], v. 49, n. 2, p. 189-196, 2019.

World Health Organization. The top 10 causes of death. Disponivel em: https://www.who.int/news-room/fact-sheets/detail/the-top-10-causes-of-death. Acesso: 23/03/2021. 